\title{
A New Method for Designing FIR Filters With Variable Characteristics
}

\author{
S. C. Chan, Member, IEEE, Carson K. S. Pun, Student Member, IEEE, and K. L. Ho, Member, IEEE
}

\begin{abstract}
This letter proposes a new method for designing finite-impulse response (FIR) filters with variable characteristics. The impulse response of the variable digital filter (VDF) is parameterized as a linear combination of functions in the spectral or tuning parameters. Using the least square objective function, the optimal solution is obtained by solving a system of linear equations. Design results show that this method is simple and effective in designing FIR VDF with good frequency characteristics. Furthermore, by using piecewise polynomial, instead of ordinary polynomial, more complicated frequency characteristics, or larger tuning range can be approximated.
\end{abstract}

Index Terms-Finite-impulse response (FIR) filters, least squares design, piecewise polynomial approximation, variable digital filter.

\section{INTRODUCTION}

$\mathbf{V}$ ARIABLE digital filters (VDFs) are digital filters with controllable spectral characteristics such as variable cutoff frequency response, adjustable passband width, controllable fractional delay, etc. They found applications in different areas of signal processing and communications, e.g., fractional delay digital filters for timing adjustment in digital receivers. Methods for designing variable digital filters can broadly be classified into two categories: transformation [1], [2], and spectral parameter approximation [4]-[6], [8]-[10] methods. In the former, a prototype filter with certain desirable frequency characteristics is first designed. Certain transformation such as the allpass transformation method [3] is then applied to the prototype filter to obtain the final VDF. In general, transformation method is applicable to VDF with variable cutoff frequencies, but not general variable characteristics say variable fractional delay. The spectral parameter approximation method is more general in that it assumes that either the impulse responses [4] or the poles and zeros [7], [9] of the filters are polynomials of certain spectral parameters. The coefficients of the polynomials are then determined to provide continuous tuning of the VDF by the spectral parameters. The spectral parameter method was proposed by Zarour and Fahmy [7], where the poles and zeros of an infinite-impulse response (IIR) filter are assumed to be polynomials of the spectral or tuning parameters. Most of the works on VDF reported are focused on the design of IIR VDF (see [4] and [9] and references therein), and methods for guaranteeing their stability [9]. More recently, the design of one-dimensional [9] and two-dimensional (2-D) [5],

\footnotetext{
Manuscript received April 26, 2002; revised June 16, 2002. The associate editor coordinating the review of this manuscript and approving it for publication was Dr. Elias S. Manolakos.

The authors are with the Department of Electrical and Electronic Engineering, The University of Hong Kong, Hong Kong (e-mail: scchan@eee.hku.hk; kspun@eee.hku.hk; klho@eee.hku.hk).

Digital Object Identifier 10.1109/LSP.2003.821686
}

[10] FIR VDF (by parameterizing the impulse response as polynomials) have received considerably attention due to their simple design procedure and good filter performance. Also, the close link between the Farrow-based fractional delay digital filter and such FIR VDF becomes more apparent [5]. This letter generalizes this concept by using a linear combination of basis functions, which can be more general functions than just polynomials. It is shown that the optimal LS solution can also be obtained by solving a system of linear equations. This differs from the weighted least squares approach in [9] and [10] in that 1) no discretization of the tuning and frequency variables are used, which helps to reduce the design complexity by means of closed-form formulas; 2 ) the approximation function is assumed to be a linear combination of basis functions. Without loss of generality, a tunable filter using a piecewise polynomial is used as an example. Design results show the LS formulation yields tunable FIR VDF with good frequency characteristics and the piecewise polynomial-based VDF offers larger tuning range than ordinary polynomial-based approach. This letter is organized as follows. In Section II, the proposed least squares design method is described. Two design examples are then given in Section III. Conclusions of this work are drawn in Section IV.

\section{Proposed LS Design Method}

The impulse response of the variable FIR filter under consideration $h(n, \boldsymbol{\Phi})$ is assumed to be a linear combination of some functions $\psi_{m}(\boldsymbol{\Phi})$ of the spectral parameters $\boldsymbol{\Phi}$, i.e.,

$$
h(n, \boldsymbol{\Phi})=\sum_{m=0}^{M-1} c_{n, m} \psi_{m}(\boldsymbol{\Phi})
$$

where $c_{m, n}$ is the coefficient of expansion. The functions $\psi_{m}(\boldsymbol{\Phi})$ can be chosen as an orthonormal basis or other functions, depending on the application at hand. Our objective is to determine $c_{m, n}$ given $\psi_{m}(\boldsymbol{\Phi})$ so that the frequency response of $h(n, \boldsymbol{\Phi})$ will approximate some desirable variable frequency response as a function of $\boldsymbol{\Phi}$, the tuning parameter vector (e.g., delay, etc.). First of all, let us consider the $z$-transform of the VDF as follows

$$
H(z, \boldsymbol{\Phi})=\sum_{n=0}^{N-1} h(n, \boldsymbol{\Phi}) z^{-n}=\sum_{n=0}^{N-1} \sum_{m=0}^{M-1} c_{n, m} \psi_{m}(\boldsymbol{\Phi}) z^{-n} .
$$

Interchanging the order of summation, (2) can be rewritten as

$$
H(z, \boldsymbol{\Phi})=\sum_{m=0}^{M-1}\left[\sum_{n=0}^{N-1} c_{n, m} z^{-n}\right] \psi_{m}(\boldsymbol{\Phi})=\sum_{m=0}^{M-1} C_{m}(z) \cdot \psi_{m}(\boldsymbol{\Phi}) .
$$


This suggests the general structure for its implementation as shown in Fig. 1. It can also be viewed as a generalization of the Farrow structure for implementing fractional delay digital filter where $h(n, \phi)$ is approximated by a polynomial in the delay parameter $\boldsymbol{\Phi}=\phi$, i.e., $\psi_{m}(\phi)=\phi^{m}$. If $H_{I}\left(e^{j \omega}, \boldsymbol{\Phi}\right)$ is the desired frequency response, the approximation error is

$$
E(\omega, \boldsymbol{\Phi})=H_{I}\left(e^{j \omega}, \boldsymbol{\Phi}\right)-\sum_{m=0}^{M-1} \sum_{n=0}^{N-1} c_{n, m} \psi_{m}(\boldsymbol{\Phi}) e^{-j n \omega} .
$$

It can be seen that $E(\omega, \boldsymbol{\Phi})$ is a linear function of the expansion coefficients $c_{m, n}$. The error cost function of $E(\omega, \boldsymbol{\Phi})$ will therefore be a quadratic function of $c_{m, n}$, which has a unique minimum characterized by a system of linear equation. More precisely, the error cost function of $E(\omega, \Phi)$ is given by

$$
\begin{aligned}
E(\omega, \boldsymbol{\Phi})= & \iint_{\mathbf{\Phi}_{S} \Omega_{S}} W\left(e^{j \omega}, \boldsymbol{\Phi}\right) \cdot|E(\omega, \boldsymbol{\Phi})|^{2} d \omega d \boldsymbol{\Phi} \\
= & \int_{\boldsymbol{\Phi}_{S} \Omega_{S}} W\left(e^{j \omega}, \boldsymbol{\Phi}\right) \\
& \cdot\left|H_{I}\left(e^{j \omega}, \boldsymbol{\Phi}\right)-\sum_{m=0}^{M-1 N-1} \sum_{n=0} c_{n, m} \psi_{m}(\boldsymbol{\Phi}) e^{-j n \omega}\right|^{2} d \omega d \boldsymbol{\Phi}
\end{aligned}
$$

where $W\left(e^{j \omega}, \boldsymbol{\Phi}\right)$ is a positive weighting function used to control the amount of approximation error in the frequency and the tuning space. The set $\Omega_{S}$ is the frequency support over which $H_{I}\left(e^{j \omega}, \boldsymbol{\Phi}\right)$ is to be approximated. For example, it can be the passband and stopband ripples of a variable cutoff digital filter. Similarly, the set $\Phi_{S}$ is the parameter space over which the spectral parameter vector $\boldsymbol{\Phi}$ is to be varied. To simplify notation, letting $l=n+N m$ and $z=e^{j \omega}$ in (2), one gets

$$
H\left(e^{j \omega}, \boldsymbol{\Phi}\right)=\sum_{m=0}^{M-1} \sum_{n=0}^{N-1} c_{n, m} \psi_{m}(\boldsymbol{\Phi}) e^{-j n \omega}=\sum_{l-0}^{N M-1} a_{l} \phi_{l}(\omega, \boldsymbol{\Phi})
$$

where $a_{l}=c_{n, m}$ with $l=n+N m$ and $\phi_{l}(\omega, \boldsymbol{\Phi})=$ $\psi_{m}(\boldsymbol{\Phi}) e^{-j n \omega}$. Substituting (5) into (4) and simplifying gives

$$
E=\boldsymbol{a}^{T} \boldsymbol{Q} a-2 \boldsymbol{b}^{T} \boldsymbol{a}+c
$$

where

$$
\begin{aligned}
\boldsymbol{a} & =\left[\begin{array}{llll}
a_{0} & a_{1} & \cdots & a_{N M-1}
\end{array}\right]^{T} \\
\boldsymbol{b} & =\left[\begin{array}{llll}
b_{0} & b_{1} & \cdots & b_{N M-1}
\end{array}\right]^{T} \\
{[\boldsymbol{Q}]_{i j} } & =\int_{\boldsymbol{\Phi}_{S}} \int_{\Omega_{S}} W\left(e^{j \omega}, \boldsymbol{\Phi}\right) \cdot \phi_{i}(\omega, \boldsymbol{\Phi}) \overline{\phi_{j}(\omega, \boldsymbol{\Phi}) \cdot} d \omega d \boldsymbol{\Phi} \\
{[\boldsymbol{b}]_{l} } & =\int_{\boldsymbol{\Phi}_{S}} \int_{\Omega_{S}} W\left(e^{j \omega}, \boldsymbol{\Phi}\right) \cdot \operatorname{Re}\left\{H_{I}\left(e^{j \omega}, \boldsymbol{\Phi}\right) \overline{\phi_{l}(\omega, \boldsymbol{\Phi})}\right\} d \boldsymbol{\Phi} d \omega
\end{aligned}
$$

and

$$
c=\int_{\boldsymbol{\Phi}_{S}} \int_{\Omega_{S}} W\left(e^{j \omega}, \boldsymbol{\Phi}\right) \cdot\left|H_{I}\left(e^{j \omega}, \boldsymbol{\Phi}\right)\right|^{2} d \omega d \boldsymbol{\Phi} .
$$

Differentiating (7) with respect to $\boldsymbol{a}$ and setting the derivatives to zero, one gets the following system of linear equation and the optimal LS solution, $\boldsymbol{a}_{\mathrm{LS}}$

$$
\boldsymbol{Q} a_{\mathrm{LS}}=\boldsymbol{b} \quad \boldsymbol{a}_{\mathrm{LS}}=Q^{-1} \boldsymbol{b} .
$$

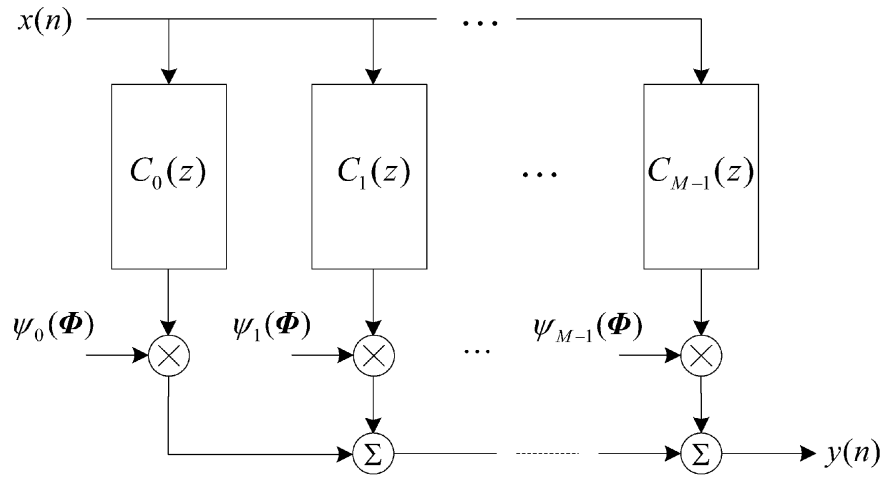

Fig. 1. General structure for implementing the proposed variable FIR digital filter.

As an illustration, let us consider the design of a lowpass FIR filter with variable cutoff frequency. The passband cutoff frequencies $\omega_{p}$ and the stopband cutoff frequency $\omega_{s}$ are assumed to vary linearly with $\boldsymbol{\Phi}=\phi$ as shown in the following:

$$
\begin{aligned}
& \omega_{p}(\phi)=\phi \cdot\left(\omega_{p 2}-\omega_{p 1}\right)+\omega_{p 1} \\
& \omega_{s}(\phi)=\phi \cdot\left(\omega_{s 2}-\omega_{s 1}\right)+\omega_{s 1} \quad\left\{\Phi_{S}: \phi \in[0,1]\right\} .
\end{aligned}
$$

Therefore, the frequency support of the filter and the desired response are respectively

$$
\begin{aligned}
& \Omega_{S}=\Omega_{p} \cup \Omega_{s}, \quad \Omega_{p}=\left\{\omega: \omega \in\left(0, \omega_{p}(\phi)\right)\right\}, \\
& \Omega_{s}=\left\{\omega: \omega \in\left(\omega_{s}(\phi), \pi\right)\right\}
\end{aligned}
$$

and

$$
H_{I}\left(e^{j \omega}, \phi\right)= \begin{cases}e^{-j \tau \omega}, & |\omega| \leq \omega_{p}(\phi) \\ 0, & \omega_{s}(\phi) \leq|\omega| \leq \pi\end{cases}
$$

where $\tau$ is the group delay which is a constant. If $h(n, \phi)$ is approximated by a polynomial, then the function $\psi_{m}(\phi)$ is just $\phi^{m}$. Putting the weighting function

$$
W\left(e^{j \omega}, \boldsymbol{\Phi}\right)= \begin{cases}K_{p} & \omega \in S_{p} \\ K_{s} & \omega \in S_{s}\end{cases}
$$

into (7), one gets

$$
\begin{gathered}
{[Q]_{i j}=\int_{0}^{1} \phi^{l+m}\left[\int_{\Omega_{p}} K_{p} e^{j(n-k) \omega} d \omega+\int_{\Omega_{s}} K_{s} e^{j(n-k) \omega} d \omega\right] d \phi} \\
=\int_{0}^{1} \phi^{l+m}\left[\int_{0}^{\omega_{p}(\phi)} 2 K_{p} \cos ((n-k) \omega) \cdot d \omega\right. \\
\left.+\int_{\omega_{s}(\phi)}^{\pi} 2 K_{s} \cos ((n-k) \omega) \cdot \omega\right] d \phi \\
=2 \int_{0}^{1} \phi^{l+m}\left[K_{p} \operatorname{sinc}\left((n-k) \omega_{p}(\phi)\right)\right. \\
+K_{s}\{\operatorname{sinc}((n-k) \pi) \\
\left.\left.\left.-\operatorname{sinc}(n-k) \omega_{s}(\phi)\right)\right\}\right] d \phi
\end{gathered}
$$




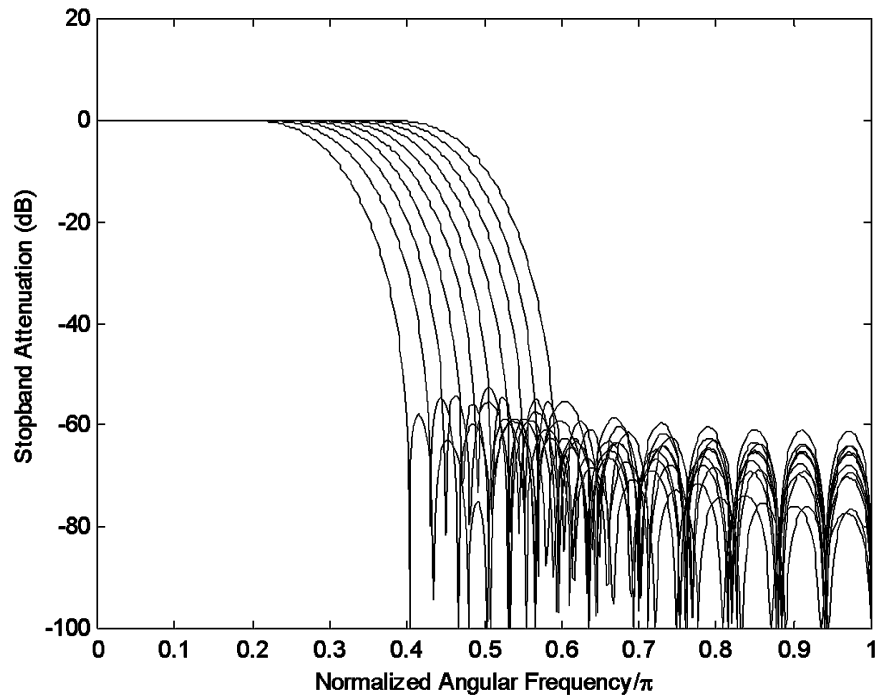

(a)

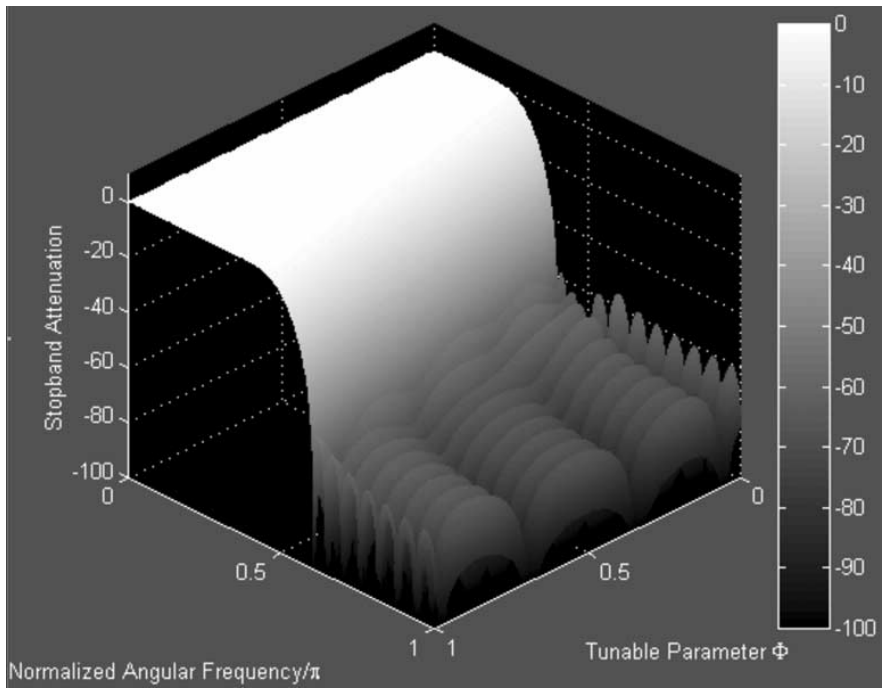

(b)

Fig. 2. Frequency response of VDF in Example 1 (a) at ten equally spaced values of $\phi$. (b) Three-dimensional plot of the variable digital lowpass filter.

and

$$
\begin{aligned}
{[\boldsymbol{b}]_{j} } & =\int_{0}^{1} \phi^{m}\left[\int_{\Omega_{p}} K_{p} \operatorname{Re}\left\{e^{j(n-\tau) \omega}\right\} d \omega\right] d \phi \\
& =2 K_{p} \int_{0}^{1} \phi^{m} \omega_{p}(\phi) \operatorname{sinc}\left((n-\tau) \omega_{p}(\phi)\right) d \phi
\end{aligned}
$$

where $i=k+N l$, and $j=n+N m$. Equations (12) and (13) can readily be calculated by the reduction formula or in general numerical integration. The optimal weighted least square solution can then be calculated from (8). The design of other variable digital filters such as variable bandpass filters and 2-D VDFs can be derived similarly. We now consider some design examples.

\section{Design EXAMPLeS}

Example 1: A lowpass tunable filter with frequency characteristics governed by (9) to (10) is designed with the following

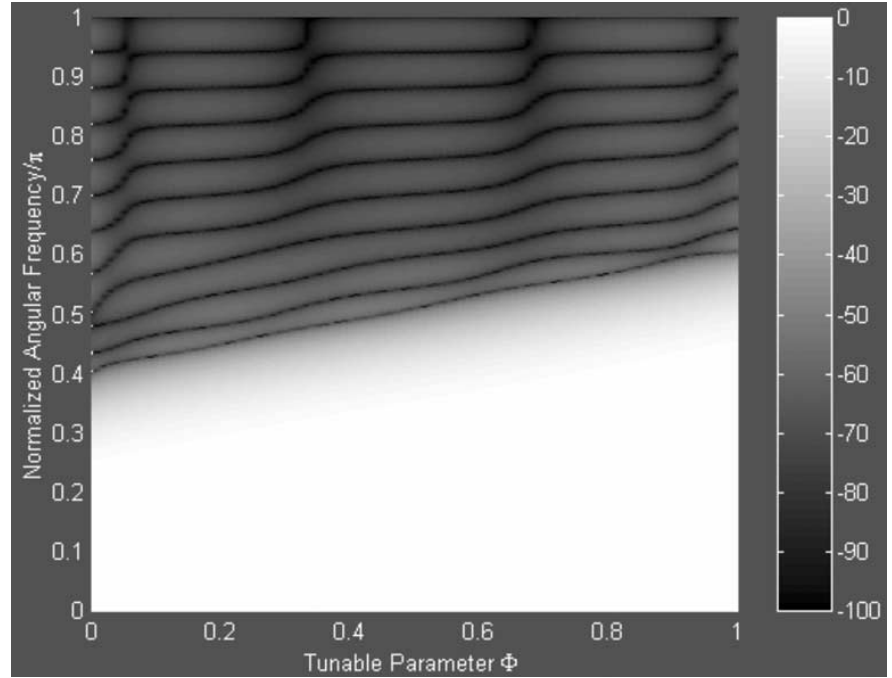

(a)

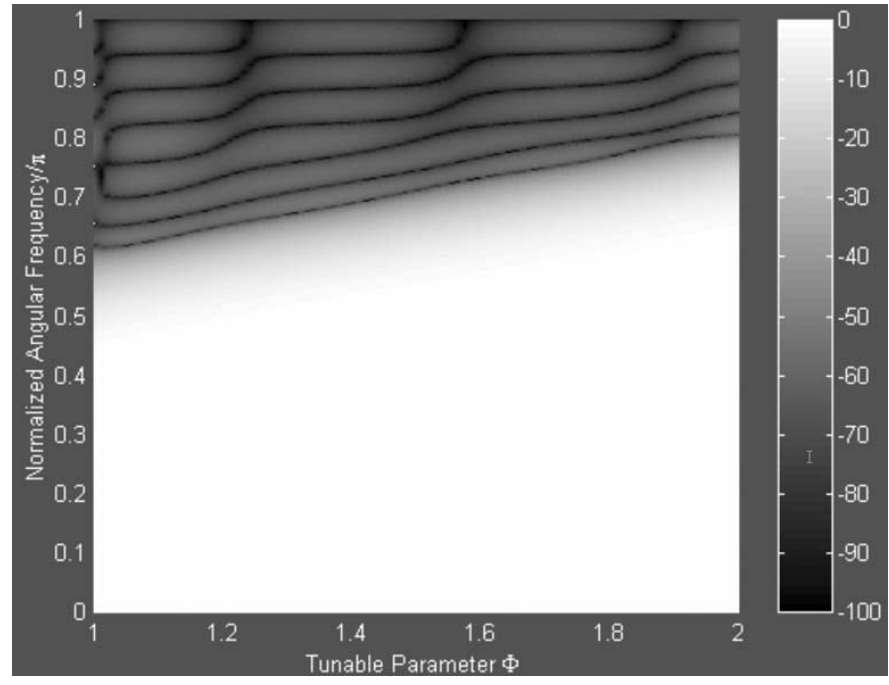

(b)

Fig. 3. Two-dimensional contour plot of the variable lowpass filter. (a) Example 1. (b) Example 2. The bar on the right shows the mapping between the grayscale and the stopband attenuation in decibels.

specification: filter length $N=32$; number of FIR subfilters $M=6$ (polynomial order is 5); tuning range: $\omega_{p 2}=0.4 \pi$, $\omega_{p 1}=0.2 \pi, \omega_{s 2}=0.6 \pi, \omega_{s 1}=0.4 \pi$. This is identical to the variable multiplierless tunable lowpass filter considered in [5], for the sake of comparison. Fig. 2(a) and (b) show, respectively, the frequency responses at ten equally spaced points of $\phi$ between 0 and 1 and its three-dimensional plot as a function of $\phi$ and $\omega$. Its 2-D contour plot is shown in Fig. 3(a). It can be seen that although the tunable range is rather large, the overall stopband attenuation is still very high over the entire tuning range. The worst case stopband attenuation of this filter is $42.885 \mathrm{~dB}$, which is roughly equal to $43.03 \mathrm{~dB}$ that was obtained in [5]. The latter is obtained by designing a large numbers of FIR filters at a dense grid of $\phi$ using the Remez exchange algorithm and a minimax criterion. The coefficients $c_{m, n}$ of the polynomials are then obtained by interpolating $h(n, \phi)$ followed by a random search for their canonical signed digit (CSD) representation. At higher frequencies, the stopband attenuation of the proposed LS tunable filter rolls off more quickly due to the use 
of the least squares objective function. This demonstrates the usefulness of the proposed approach and its simple design using the closed-form formula.

Example 2: In this example, we are going to extend the tuning range of example 1 to $\omega_{p 2}=0.6 \pi, \omega_{p 1}=0.4 \pi$, $\omega_{s 2}=0.8 \pi, \omega_{s 1}=0.6 \pi$ by treating $h(n, \phi)$ as a piecewise polynomial. More precisely, $h(n, \phi)$ is approximated by the structure obtained in example 1 in the range $\omega_{p 2}=0.4 \pi$, $\omega_{p 1}=0.2 \pi, \omega_{s 2}=0.6 \pi, \omega_{s 1}=0.4 \pi$ when $\phi$ varies from 0 to 1. $h(n, \phi)$ in the extended tuning range is approximated by another polynomial when $\phi$ varies from 1 to 2 . Only one of these two structures will be active at the same time, depending on the tuning range. For simplicity, we do not impose smoothing constraints between this piecewise polynomial. In other words, they can be designed separately using the proposed method in Section II. Fig. 3(b) shows the VDF in the extended tuning range, which demonstrates the usefulness of the piecewise polynomial approach in extending the tuning range of the VDF.

\section{CONCLUSION}

A new method for designing FIR filters with variable characteristics is presented. It parameterizes the impulse response of the variable digital filter as a linear combination of functions in the spectral or tuning parameters. This allows closed-form optimal least squares solution to be obtained by solving a system of linear equations. Design results show the LS formulation yields tunable FIR VDF with good frequency characteristics and the piecewise polynomial-based VDF offers larger tuning range than ordinary polynomial-based approach.

\section{ACKNOWLEDGMENT}

The authors would like to thank T. B. Deng for providing us with his papers on variable digital filter designs.

\section{REFERENCES}

[1] A. V. Oppenheim, W. F. G. Mecklenbrauker, and R. M. Mersereau, "Variable cutoff linear phase digital filters," IEEE Trans. Circuits Syst., vol. 23, pp. 199-203, Apr. 1976.

[2] P. Jarske, Y. Neuvo, and S. K. Mitra, "A simple approach to the design of linear phase FIR digital filters with variable characteristics," Signal Process., vol. 14, no. 4, pp. 313-326, June 1988.

[3] A. G. Constantinides, "Spectral transformation for digital filters," Proc. Inst. Elect. Eng., pp. 1585-1590, 1970.

[4] T. B. Deng and T. Soma, "Variable digital filter design using the outer product expansion," in Proc. Inst. Elect. Eng. Vision, Image Signal Process., vol. 141, Apr. 1994, pp. 123-128.

[5] C. K. S. Pun, S. C. Chan, and K. L. Ho, "Efficient 1D and circular symmetric 2D FIR filters with variable cutoff frequencies using the Farrow structure and multiplier-block," in Proc. ISCAS, vol. 2, pp. 561-564.

[6] C. W. Farrow, "A continuously variable digital delay element," in Proc. ISCAS, 1988, pp. 2641-2645.

[7] R. Zarour and M. M. Fahmy, "A design technique for variable digital filters," IEEE Trans. Circuits Syst. II, vol. 36, pp. 1473-1478, Nov. 1989.

[8] A. Tarczynski, G. D. Cain, E. Hermanowicz, and M. Rojewski, "WLS design of variable frequency response FIR filters," in Proc. ISCAS, vol. 4, 1997, pp. 2244-2247.

[9] T. B. Deng, "Design of recursive 1-D variable filters with guaranteed stability," IEEE Trans. Circuits Syst. II, vol. 9, pp. 689-695, Sept. 1997.

[10] —, "Variable 2-D FIR digital filter design and parallel implementation," IEEE Trans. Circuits Syst. II, vol. 46, pp. 631-635, May 1999. 